International Journal of Medical Anesthesiology 2020; 3(4): 01-06

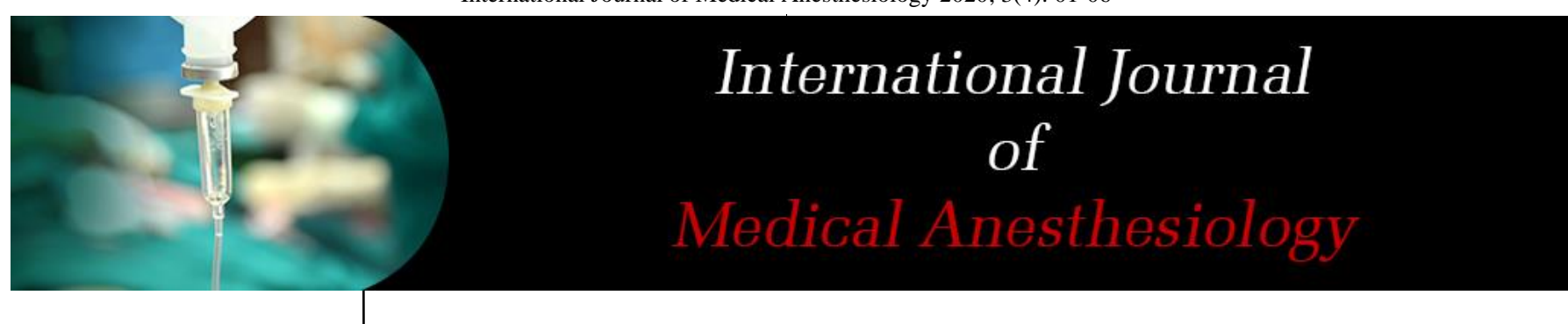

E-ISSN: 2664-3774

P-ISSN: 2664-3766 www.anesthesiologypaper.com IJMA 2020; 3(4): 01-06

Received: 01-08-2020

Accepted: 03-09-2020

Dr. Anupama MK Assistant Professor Department of Anesthesiology, PKDAS Institute of Medical Sciences, Vaniamkulam, Ottapalam, Kerala, India

\section{Attenuation of haemodynamic responses by dexmedetomidine during laryngoscopy and intubation: A double blinded randomized controlled study}

\section{Dr. Anupama MK}

DOI: https://doi.org/10.33545/26643766.2020.v3.i4a.156

\begin{abstract}
Background: Laryngoscopy and tracheal intubation cause hemodynamic response which cause tachycardia, hypertension and increased myocardial oxygen demand which adversely affects the patient's condition intraoperatively.

Various drugs were investigated to attenuate the hemodynamic responses caused by laryngoscopy and intubation.

A drug which can blunt both the heart rate response and blood pressure response of laryngoscopy and intubation, without having any adverse effects would be ideal.

Dexmedetomidine, a relatively newer alpha agonist, has shown to attenuate haemodynamic response to direct laryngoscopy and endotracheal intubation without much side effects.

The present study was undertaken to evaluate the effects of recently introduced alpha- 2 agonist, dexmedetomidine as premedication in attenuating pressor response during Laryngoscopy and Endotracheal intubation. This was double blind, prospective, randomized controlled study.

Material and Methods: Test group: Patients received Intravenous Dexmedetomidine $1 \mu \mathrm{g}$ per $\mathrm{kg}$ in $100 \mathrm{ml}$ normal saline infusion for $15 \mathrm{mins}$

Control group: Patients received $100 \mathrm{ml}$ normal saline infusion for $15 \mathrm{mins}$.

Results: Dexmedetomidine at a dose of $1 \mu \mathrm{g} / \mathrm{kg}$ body weight diluted in $100 \mathrm{ml}$ of normal saline given intravenously before induction significantly attenuates the haemodynamic responses to laryngoscopy and tracheal intubation.

Dexmedetomidine also decreases the requirement of induction dose of propofol.

Dexmedetomidine also decreases the total dose of vecuronium bromide required for muscle relaxation. There were no significant adverse postoperative events associated with the use of preoperative dexmedetomidine

Conclusion: Dexmedetomidine at a dose of $1 \mu \mathrm{g} / \mathrm{kg}$ body weight diluted in $100 \mathrm{ml}$ of normal saline given intravenously before induction significantly attenuates the haemodynamic responses to laryngoscopy and tracheal intubation.
\end{abstract}

Keywords: Laryngoscopy, tracheal intubation, haemodynamic responses, dexmedetomidine

\section{Introduction}

Laryngoscopy and tracheal intubation are noxious stimuli that provoke a transient but marked sympathetic response, manifesting as tachycardia and hypertension. These haemodynamic responses to laryngoscopy and tracheal intubation were first recognized as early as in 1940s by Reid and Brace et al. ${ }^{[1]}$. These facts were further confirmed by various investigators and were supposed as a result of reflex sympathoadrenal response ${ }^{[2,3]}$.

These responses are transitory, variable and are much more pronounced in hypertensive than in normotensive individuals ${ }^{[4]}$.

These haemodynamic response to laryngoscopy and tracheal intubation may not be of much significance in an otherwise normal individual, but in suspectible patients particularly those with systemic hypertension, coronary artery disease, cerebrovascular disease or intracranial aneurysms even these transient changes can result in potentially deleterious effect like LVF, pulmonary oedema, myocardial ischaemia, ventricular dysrrthymias and cerebral haemorrhage.

Robert K. Stoelting [5], noted that the best way to prevent laryngoscopic reaction was to minimize the duration of laryngoscopy and intubation. He noted that if laryngoscopy and intubation was performed within 15 seconds, the haemodynamic responses were minimal.
Dr. Anupama MK

Assistant Professor

Department of Anesthesiology,

PKDAS Institute of Medica

Sciences, Vaniamkulam,

Ottapalam, Kerala, India 
He also suggested that i.v. lignocaine given in the dose of laryngoscopy and endotracheal intubation. However many authors have noted that lignocaine fails to attenuate the haemodynamic responses to laryngoscopy and intubation effectively.

Direct acting vasodilators like sodium nitroprusside and nitroglycerine were tried for obtunding the haemodynamic responses to laryngoscopy and intubation. The authors observed that, though they were powerful in attenuating these responses, the reflex tachycardia they caused limited their usefulness in blunting the laryngoscopic response and in addition their use needed invasive arterial pressure monitoring.

Calcium channel blockers like nifedipine and verapamil were studied widely to suppress the haemodynamic responses to laryngoscopy and tracheal intubation.

As the haemodynamic response occurring during laryngoscopy and tracheal intubaton was due to augmented sympathetic response, the investigators thought that by using pharmacological drugs with specific adrenergic blocking properties, they could reduce the haemodynamic responses to laryngoscopy and tracheal intubation.

Due to its central sympatholytic effect, dexmedetomidine is useful in blunting haemodynamic responses in perioperative period. It is successfully used in intravenous doses varying from 0.25 to $1 \mathrm{mcg} / \mathrm{kg}$ for attenuating intubation response. Optimal dose for attenuating pressor response seems to be 1 $\mathrm{mcg} / \mathrm{kg}$ with lesser doses not being effective. Infusion continued into the postoperative period has been associated with reduced haemodynamic fluctuations and decrease in plasma catecholamines. Doses in the range of $0.5 \mathrm{mcg} / \mathrm{kg}$ not only blunted the extubation response but also reduced the emergence reaction and analgesic requirement to extubation following rhinoplasty and neurosurgery.

\section{Primary objective}

To evaluate the efficacy of intravenous dexmedetomidine in the dose of $1 \mu \mathrm{g} / \mathrm{kg}$ body weight in attenuating the haemodynamic responses to laryngoscopy and endotracheal intubation.

\section{Secondary objectives}

1. To study the effects of dexmedetomidine on the dose requirement of propofol for induction of anaesthesia.

2. To study the effects of dexmedetomidine on the dose requirement of vecuronium bromide for muscle relaxation.

3. To study any adverse effects associated with dexmedetomidine administration.

\section{Methods and Materials}

A double blind randomized controlled study was undertaken at Apollo BGS Hospitals, Mysore during the period December 2014 to November 2016. The study was undertaken after obtaining ethical committee clearance as well as informed consent from all patients.

One hundred adult patients of either sex, scheduled for various elective surgeries, under general anaesthesia were included in the study.

\section{Inclusion Criteria for the Study}

1. ASA- Physical Status I \& II.

2. Age between 20-40 years, both genders.

3. Elective surgeries under general anaesthesia.

\section{Exclusion Criteria for the Study}

1. Patients with anticipated difficult airway,

2. Patients on hypnotics and antidepressants,

3. H/o Hiatus Hernia, GERD, respiratory, hepatic, renal diseases,

4. $\mathrm{BMI}>30$,

5. Patients with ASA grade III and above.

The study population was randomly divided into two groups with 50 patients in each group using shuffled, sealed, opaque, envelope method, and the patient was asked to pick the envelope. The envelope was opened by the senior anaesthesiologist who would also prepare the study drug but not involved with observations of the study.

Test group: Patients will receive Intravenous Dexmedetomidine $1 \mu \mathrm{g}$ per $\mathrm{kg}$ in $100 \mathrm{ml}$ normal saline infusion for $15 \mathrm{mins}$.

Control group: Patients will receive $100 \mathrm{ml}$ normal saline infusion for 15 mins.

Preanaesthetic evaluation was done on the evening before surgery. A routine preanaesthetic examination was conducted assessing:

1. General condition of the patient.

2. Detailed Airway assessment by Mallampati classification, Patil's rule of 3-3-2, neck extension, thyromental distance and upper lip bite test.

3. Nutritional status and body weight of the patient.

4. A detailed examination of the cardiovascular system, Respiratory system, central nervous system and abdomen.

The following investigations were done in all patients
- Haemoglobin
- Blood Group
- Coagulation profile
- Blood Urea
- Serum Creatinine
- RBS/FBS
- Chest Xray

Other relevant investigations

- Thyroid Function Test

- Liver Function Test

- Neck X-ray

All patients included in the study were premedicated with Inj. Ranitidine $50 \mathrm{mg}$ and Inj. Ondansetron $4 \mathrm{mg}$ half an hour before shifting to operation theatre. They were kept nil orally for solids for at least $6 \mathrm{hrs}$ before surgery.

On the day of surgery, Anaesthesia machine and circuits were checked, resuscitation equipments were kept ready. After confirmation of NPO status, patients were shifted to the operating room and were connected to monitor. Basal systolic blood pressure (SBP), diastolic blood pressure (DBP), Mean arterial pressure (MAP), Heart rate (HR) were recorded after 5 mins of settling in the OT.

An Intravenous line was secured with $18 \mathrm{G}$ cannula and preloading with $500 \mathrm{ml}$ of Ringer lactate were done over 30 mins for all patients. Following this, 
Test group: Patients received Intravenous Dexmedetomidine $1 \mu \mathrm{g}$ per $\mathrm{kg}$ in $100 \mathrm{ml}$ normal saline infusion for $15 \mathrm{mins}$

Control group: Patients received $100 \mathrm{ml}$ normal saline infusion for $15 \mathrm{mins}$.

After 5mins, SBP, DBP, MAP, Heart rate, (T1) were recorded.

Prior to induction, Inj Glycopyrrolate $0.2 \mathrm{mg}$, Inj Midazolam $1 \mathrm{mg}$ and Inj Fentanyl 100microgm were administered IV. All patients were pre- Oxygenated with $100 \%$ Oxygen for $3 \mathrm{mins}$ and Anaesthesia were induced with $2 \mathrm{mg} / \mathrm{kg}$ Propofol $1 \%$. After confirmation of adequacy of Bag and Mask Ventilation, patients were given Vecuronium $0.1 \mathrm{mg} / \mathrm{kg}$ to facilitate laryngoscopy and intubation. At $2 \mathrm{mins}$ after induction, SBP, DBP, MAP, Heart rate were recorded (T2). With a Macintosh blade Laryngoscope, Intubation were done after 3 mins of induction with well lubricated, appropriate sized cuffed oral endotracheal tube. Confirmation of the tube position was done by bilateral auscultation for air entry and checking capnograph waveform on the monitor. SBP, DBP, MAP, Heart rate were recorded. After confirmation of the tube position and fixing it, oral endotracheal tube were connected to Anaesthesia Work Station. After adjusting appropriate ventilator settings, Anaesthesia was maintained with $\mathrm{N}_{2} \mathrm{O}$ and $\mathrm{O}_{2}$ in ratio of $70 \%: 30 \%$, lower possible concentrations of Desflurane, and Vecuronium maintenance doses IV. SBP, DBP, MAP, and Heart rate were recorded at 1 (T3), 5 (T4), and $10 \mathrm{~min}$ (T5), after laryngoscopy and intubation. Residual Neuromuscular blockade reversal were done with IV Neostigmine $(0.05 \mathrm{mg} / \mathrm{kg})$ and IV Glycopyrrolate $(0.01 \mathrm{mg} / \mathrm{kg})$.

Postoperatively, Consciousness, blood pressure, heart rate, and oxygen saturation ( $\mathrm{SpO} 2)$ were monitored. Sedation scoring at the end of surgery was done using Ramsay sedation score.

\section{Monitoring}

The following cardiovascular parameters were recorded in all patients

- $\quad$ Systolic blood pressure(SBP) in $\mathrm{mm}$ of $\mathrm{Hg}$

- Diastolic blood pressure(DBP) in $\mathrm{mm}$ of $\mathrm{Hg}$

- Mean arterial pressure(MAP) in $\mathrm{mm}$ of $\mathrm{Hg}$

- Heart rate(HR) in beats per minute

The above cardiovascular parameters were monitored at the following time interval

1. Basal Reading when patient is shifted to OT.

2. At 5 min after infusion of Dexmedetomidine /Saline.

3. At Induction (2min after Propofol + Vecuronium).

4. At $1 \mathrm{~min}$ after intubation.

5. At $5 \mathrm{~min}$ after intubation.

6. At $10 \mathrm{~min}$ after intubation.

\section{Statistical Methods Employed}

\section{Sample size calculation}

The number of participants required in each study, m, will be calculated using the formula as below:

$\mathrm{m}=\frac{2 \times[Z(1-\alpha / 2)+Z(1-\beta)]^{2}}{\Delta^{2}}$

Where $Z(1-\alpha / 2)$ and $Z(1-\beta)$ represent percentage points of the normal distribution for statistical significance level and power, respectively and $\Delta$ represents the standardized difference (i.e. the treatment difference divided by its standard deviation).

The standardised difference $\Delta=p^{1}-p^{2}$

$$
\sqrt{\mathrm{p} \times(1-p)}
$$

where $p=\frac{\left(p^{1}+p^{2}\right)}{2}$

In our study, $\mathrm{p}^{1}=0.80($ or $80 \%), \mathrm{p}^{2}=0.40($ or $40 \%)$ and so, $\mathrm{p}=\underline{0.8+0.4}$

$$
=0.6
$$

Hence, $\Delta=0.8-0.4$

$$
\begin{aligned}
& \sqrt{0.6 \times(1-0.6)} \\
= & 0.82
\end{aligned}
$$

Using the values for a significance level of $5 \%, \mathrm{z}(1-$ $\alpha / 2)=1.96$, and a power of $80 \%, z(1-\beta)=0.8416$,

$$
\begin{aligned}
\mathrm{m} & =\frac{2 \times[1.96+0.8]^{2}}{(0.82)^{2}} \\
& =23^{(2)}
\end{aligned}
$$

From the above calculations, it is found that the minimal no. of patients required for the study is 23. In order to compensate for the drop-outs and to have a better power we studied 50 patients in each.

The Independent- Samples ' $t$ ', Crosstabs, Repeated measures ANOVA were the statistical methods used.

\section{Results}

The mean age in Test Group and Control group were $34.12 \pm 6.08$ and $34.96 \pm 6.10$ respectively.

There was no significant difference in the age of patients between the Test Group and Control

Group. Both the groups were similar with respect to age distribution $(\mathrm{p}=0.492)$.

Statistically there was no significant change in the gender wise distribution of patients in both the groups ( $\mathrm{p}=0.841)$.

The mean body weight in Test group was $58.04 \pm 4.63$ and in Control Group, it was 59.02 \pm 5.06 .

There was no significant difference in the body weight of patients between the Test Group and

Control Group $(\mathrm{p}=0.848)$

Table 1: Intergroup comparison of mean heart rate (bpm) changes in response to laryngoscopy and intubation between Test Group and Control Group

\begin{tabular}{|c|c|c|c|}
\hline Time & Test Group & Control Group & p-value \\
\hline Basal & $86.04 \pm 13.85$ & $84.50 \pm 9.87$ & 0.523 \\
\hline Pre-Induction & $80.14 \pm 10.32$ & $89.04 \pm 10.52$ & 0.000 \\
\hline Induction & $71.62 \pm 10.14$ & $93.12 \pm 10.0$ & 0.000 \\
\hline 1 min & $79.38 \pm 8.54$ & $118.4 \pm 7.29$ & 0.000 \\
\hline $5 \mathrm{~min}$ & $73.74 \pm 7.75$ & $105.94 \pm 6.7$ & 0.000 \\
\hline $10 \mathrm{~min}$ & $71.78 \pm 8.26$ & $95.62 \pm 5.72$ & 0.000 \\
\hline
\end{tabular}

The basal heart rate were comparable in both groups $(p=0.523)$. Statistical evaluation between the groups showed a significant fall in Heart rate in Test Group after induction. The mean heart rate increase observed at 1,5 and 10 minutes after intubation in Control Group was statistically highly significant compared to mean heart rate in Test Group $(\mathrm{p}=0.000)$. 
Table 2: Intergroup comparison of mean systolic blood pressure ( $\mathrm{SBP}$ in $\mathrm{mmHg}$ ) changes in response to laryngoscopy and intubation between Control group and Test Group

\begin{tabular}{|c|c|c|c|}
\hline Time & Test Group & Control Group & p-value \\
\hline Basal & $126.64 \pm 7.39$ & $128.28 \pm 5.63$ & 0.155 \\
\hline Pre- Induction & $119.76 \pm 7.28$ & $130.82 \pm 6.15$ & 0.000 \\
\hline Induction & $101.64 \pm 4.04$ & $128.52 \pm 6.53$ & 0.000 \\
\hline 1 min & $110.32 \pm 3.89$ & $155.26 \pm 7.73$ & 0.000 \\
\hline 5 min & $105.38 \pm 4.64$ & $140.54 \pm 9.80$ & 0.000 \\
\hline 10 min & $104.48 \pm 4.24$ & $130.28 \pm 8.32$ & 0.000 \\
\hline
\end{tabular}

$(p<0.01)$ - Highly significant (HS); $(p<0.05)$ - Significant (S); $(p>0.05)$ - Not significant(NS)

The mean systolic blood pressure were comparable in both groups $(\mathrm{p}=0.155)$. The mean systolic blood pressure values after induction were significantly low $(\mathrm{p}=0.000)$ compared to Control

Group. The increase in systolic blood pressure in Control Group at 1, 5 and 10 minutes after intubation was statistically highly significant $(\mathrm{p}=0.000)$ compared to Test Group.

Table 3: Intergroup comparison of mean diastolic pressure (DBP in $\mathrm{mmHg}$ ) changes in response to laryngoscopy and intubation between Control group and Test group

\begin{tabular}{|c|c|c|c|}
\hline Time & Test Group & Control Group & p-value \\
\hline Basal & $79.04 \pm 4.94$ & $77.04 \pm 6.15$ & 0.076 \\
\hline Pre- Induction & $74.88 \pm 5.20$ & $80.8 \pm 6.44$ & 0.000 \\
\hline Induction & $69.52 \pm 3.09$ & $78.20 \pm 6.88$ & 0.000 \\
\hline 1 min & $74.24 \pm 3.08$ & $98.8 \pm 3.45$ & 0.000 \\
\hline 5 min & $71.10 \pm 3.28$ & $86.10 \pm 8.61$ & 0.000 \\
\hline 10 min & $69.96 \pm 2.79$ & $80.48 \pm 7.48$ & 0.000 \\
\hline
\end{tabular}

$(p<0.01)$ - Highly significant (HS); $(p<0.05)$ - Significant $(\mathrm{S})$; $(p>0.05)$ - Not significant(NS)

The mean diastolic blood pressure were comparable in both the groups $(p=0.076)$. The increase in diastolic blood pressure in Control Group at 1,5 and 10 minutes after intubation were statistically highly significant $(\mathrm{p}=0.000)$ compared to Test Group.

Table 4: Intergroup comparison of mean arterial pressure (MAP in $\mathrm{mmHg}$ ) changes in response to laryngoscopy and intubation between Control group and Test group

\begin{tabular}{|c|c|c|c|}
\hline Time & Test Group & Control Group & p-value \\
\hline Basal & $92.28 \pm 5.15$ & $93.80 \pm 5.17$ & 0.145 \\
\hline Pre- Induction & $86.88 \pm 4.17$ & $97.24 \pm 5.37$ & 0.000 \\
\hline Induction & $81.20 \pm 2.91$ & $93.92 \pm 5.80$ & 0.000 \\
\hline 1 min & $85.96 \pm 3.39$ & $118.04 \pm 3.41$ & 0.000 \\
\hline 5 min & $83.62 \pm 3.18$ & $103.42 \pm 7.37$ & 0.000 \\
\hline 10 min & $82.52 \pm 4.10$ & $96.44 \pm 6.07$ & 0.000 \\
\hline
\end{tabular}
$(p<0.01)$ - Highly significant (HS); $(p<0.05)$ - Significant (S); $(p>0.05)$ - Not significant(NS)

The mean basal mean arterial pressure were comparable in both the groups $(\mathrm{p}=0.145)$. There was a significant difference in mean arterial pressure value after induction, which was statistically highly significant $(p=0.000)$. The increase in mean arterial pressure in Control Group was statistically highly significant at 1,5 and 10 minutes after intubation ( $\mathrm{p}=0.000)$ compared to Test Group.

The mean dose of propofol required for loss of eye lash reflex in Test group and Control Group were 98.69 \pm 13.98 $\mathrm{mg}$ and $114.66 \pm 5.35 \mathrm{mg}$ respectively. Statistical evaluation between the groups showed a statistically highly significant reduction in the dose of propofol required for induction $(\mathrm{p}=0.000)$ in Test Group.

The mean dose of vecuronium bromide required for muscle relaxation in Test Group and Control Group were 4.28 \pm 0.47 $\mathrm{mg}$ and $5.34 \pm 0.82 \mathrm{mg}$ respectively. Statistical evaluation between the groups showed a statistically significant reduction in the dose of vecuronium bromide required for muscle relaxation $(\mathrm{p}=0.000)$ in Test Group.

In Test Group, mean sedation score immediately after extubation is $2.68 \pm 0.59$ (Responding to commands) and in Control Group mean sedation score is $2.38 \pm 0.70$ (Cooperative, oriented and tranquil). Statistical evaluation between the groups showed that the patients in Test Group were more sedated than the patients in Control Group which is statistically significant $(\mathrm{p}=0.021)$, but clinically nonsignificant.

\section{Discussion}

Direct laryngoscopy and endotracheal intubation form the mainstay of general anaesthesia. Laryngoscopy and endotracheal intubation are considered as the most critical events during general anaesthesia as they provoke transient but marked sympathoadrenal response manifesting as hypertension and tachycardia ${ }^{4}$.

These responses are transient, variable and may not be significant in normal individuals. But in patients with cardiovascular diseases like hypertension, ischemic heart disease, cerebrovascular disease, intracranial aneurysms, these transient changes in haemodynamic variables can result in potentially harmful effects like left ventricular failure, pulmonary oedema, myocardial ischemia and cerebral haemorrhage. It becomes essential to attenuate this sympathetically driven haemodynamic response to laryngoscopy and endotracheal intubation ${ }^{6}$

Many methods have been developed to attenuate or prevent this haemodynamic response like deepening the plane of anaesthesia, using lidocaine, using topical anaesthesia, omitting atropine as a premedicant, premedicating with Nitroglycerin, Betablockers, calcium channel blockers, opiods, gabapentin, pregabalin. The latest entrants to this group are Alpha-2 agonists. Alpha-2 agonists have been used for attenuating the sympathetic response and among $\alpha$ 2 agonists both clonidine and dexmedetomidine have been used. Dexmedetomidine is highly specific and selective $\alpha-2$ adrenoceptor agonist with $\alpha 2: \alpha 1$ binding selectivity ratio of 1620:1 compared to 220:1 for clonidine.

These drugs have their own limitations like opiods cause respiratory depression and chest wall rigidity, halothane causes dysrrhythmias, calcium channel blockers produce reflex tachycardia, direct acting vasodilators produce precipitous fall in blood pressure and hence needing invasive haemodynamic monitoring. Beta blockers blunt the heart rate response better than blood pressure response $\mathrm{e}^{7,8}$.

A drug which can blunt both the heart rate response and blood pressure response of laryngoscopy and intubation, without having any adverse effects would be ideal.

Dexmedetomidine, a relatively newer alpha agonist, has shown to attenuate haemodynamic response to direct laryngoscopy and endotracheal intubation without much side effects.

The present study was undertaken to know the efficacy of dexmedetomidine in blunting the haemodynamic response to laryngoscopy and intubation in a double blind, prospective randomized controlled study. 
Gandhi S et al. in $2014^{\text {[9] }}$ compared dexmeditomedine 0.6 $\mathrm{mcq} / \mathrm{kg}$ and fentanyl $2 \mathrm{mcq} / \mathrm{kg}$ in suppressing the pressor response and concluded that DEXMEDETOMIDINE when used as I.V. premedicant in dose of $0.6 \mu \mathrm{g} / \mathrm{kg}$ provides beneficial effect in attenuation of pressor response to laryngoscopy and endotracheal intubation as compare to FENTANYL in dose of $2 \mu \mathrm{g} / \mathrm{kg}$.

Reddy SV et al. in $2014^{[10]}$ studied the effects of dexmedetomidine with esmolol and control in attenuating the presser response during laryngoscopy and concluded that dexmedetomidine $1.0 \mu \mathrm{g} / \mathrm{kg}$ provides a consistent, reliable and effective attenuation of pressure responses when compared to esmolol $2 \mathrm{mg} / \mathrm{kg}$.

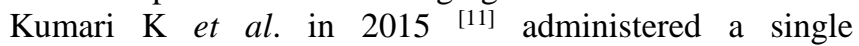
preinduction intravenous dose of dexmedetomidine of 0.5 $\mathrm{mg} / \mathrm{kg}$ which resulted in significant attenuation of the rise in the heart rate, systolic blood pressure, diastolic blood pressure, and mean blood pressure, until 5 minutes postintubation and significantly reduced the dose requirements of propofol for induction and caused minimal side effects

El-Shmaa NS et al. in $2016^{[12]}$ studied that Dexmedetomidine attenuates the hemodynamic stress response to laryngoscopy and intubation more effectively compared with labetalol without any deleterious effects. Furthermore, dexmedetomidine decreased the dose of propofol for induction of anesthesia as guided by bispectral index.

Pokhrel N et al. $2016^{[13]}$, in their study on, "Attenuation of hemodynamic response to laryngoscopy and endotracheal intubation with dexmedetomidine: a randomized controlled trial".

Rachit B et al. in 2016 [14], studied "Evaluation of Dexmedetomidine- $0.5 \mu \mathrm{g} / \mathrm{kg}$ and $1 \mu \mathrm{g} / \mathrm{kg}$ in Blunting the Responses to Laryngoscopy and Intubation". They found that dexmedetomidine in a loading dose of $1 \mu \mathrm{g} / \mathrm{kg}$ body weight significantly attenuates response to laryngoscopy and intubation with minimal incidence of side effects as compared with dexmedetomidine in a dose of $0.5 \mu \mathrm{g} / \mathrm{kg}$ which was found insufficient in majority of cases to cause complete attenuation of sympathetic response.

\section{Limitations of the Study}

1. We did not use neuromuscular monitor for accurately measuring the required dose of vecuronium bromide, due to non-availability of the monitor in our hospital. This may modify the accuracy of the total dose of vecuronium bromide calculated in both the groups.

2. Since Dexmedetomidine also is known to have analgesic properties and has post operative sparing effects, we did not find out the same in our study, as it was not in our objectives.

3. The interactions of dexmedetomidine with other drugs and anaesthetic agents were not studied.

4. Co-morbid disease states were excluded from this study, where suppression of haemodynamic response is a pre-requisite

\section{Conclusion}

1. Dexmedetomidine at a dose of $1 \mu \mathrm{g} / \mathrm{kg}$ body weight diluted in $100 \mathrm{ml}$ of normal saline given intravenously before induction significantly attenuates the haemodynamic responses to laryngoscopy and tracheal intubation.
2. Dexmedetomidine also decreases the requirement of induction dose of propofol.

3. Dexmedetomidine also decreases the total dose of vecuronium bromide required for muscle relaxation.

4. There were no significant adverse postoperative events associated with the use of preoperative dexmedetomidine.

\section{Recommendations}

1. We recommend the use of neuromuscular monitoring during the study to know the exact requirement of neuromuscular blocking agent.

2. Use of pain scoring systems to assess the post-operative pain, as dexmedetomidine has analgesic effect and opiod sparing property.

3. Studies on interaction of dexmedetomidine with other drugs and anaesthetic agents needs to be done.

4. The effect of dexmedetomidine in attenuating the haemodynamic response to direct laryngoscopy and endotracheal intubation in population with comorbid disease states like Hypertension, Diabetes Mellitus needs to be studied as they are more vulnerable for complications due to this response.

5. Comparison of other agents (like Clonidine, Pregablin, etc) with dexmedetomidine for their efficacy in suppressing intubation response.

\section{References}

1. Reid LC, Brace DE. Irritation of respiratory tract \& its relex effect effect upon heart. Surg Gynae obstet 1940;70:157-62.

2. Kayhan Z, Aldemir D, MEtler H, Ogus E. Which is responsible for the haemodynamic response due to laryngoscopy and endotracheal intubation? Cathecholamines, vasopressin or angiotensin? European Journal of Anaesthesiology 2005;22:780-5.

3. Morin AM, Gelbner G, Schwarz U, Kahl M et al. Factors influencing preoperative stress responses in coronary artery bypass graft patients. BMC anaesthesiology, 2004, 4(7).

4. Prys-Roberts C, Gressn LT, Meloche R, Foex P. Studies of anesthesia in relation to hypertension haemodynamic consequences of induction and endotracheal intubation. British Journal of Anaesthesia 1971;43:531-47.

5. Stoelting RK. Circulatory changes during laryngoscopy $\&$ intubation influence of duration of laryngoscopy with or without lidocaine. Anesthesiology 1977; 47:381-4.

6. King BD, Harris LC, Greifenstein FE, Elderb JD, Dripps RD. Reflex circulatory responses to direct laryngoscopy \& tracheal intubation performed during general anesthesia. Anesthesiology, 1951, 556-66.

7. Prys- Roberts C, Foex P, Biro GP. Studies of anaesthesia in relation to hypertension versus adrenergic $\beta$ receptor blockade. British Journal of Anaesthesia, 1973;45:671-80.

8. McCammon RL, Hilgenberg JC, Stoelting RK. Effect of Propanolol on circulatory responses to induction of diazepam- nitrous oxide anaesthesia and to endotracheal intubation. Anesthesia \& Analgesia 1981; 60(8):579-83.

9. Gandhi S, Goyal V, Radhakrishnan K, Balakrishnan M. Comparison of Dexmedetomidine with Fentanyl in Attenuation of Pressor Response during Laryngoscopy 
and Intubation. IOSR Journal of Pharmacy 2014;2(4):28-38.

10. Reddy SV, Balaji D, Ahmed SN. Dexmedetomidine versus esmolol to attenuate the hemodynamic response to laryngoscopy and tracheal intubation: A randomized double-blind clinical study. International Journal of Applied and Basic Medical Research 2014;4(2):95.

11. Kumari K, Gombar S, Kapoor D, Sandhu HS. Clinical study to evaluate the role of preoperative dexmedetomidine in attenuation of hemodynamic response to direct laryngoscopy and tracheal intubation. Acta Anaesthesiologica Taiwanica 2015;53(4):123-30.

12. El-Shmaa NS, El-Baradey GF. The efficacy of labetalol vs dexmedetomidine for attenuation of hemodynamic stress response to laryngoscopy and endotracheal intubation. Journal of clinical anesthesia 2016;31:26773.

13. Pokhrel N, Bajracharya UB. Attenuation of hemodynamic response to laryngoscopy and endotracheal intubation with dexmedetomidine: a randomized controlled trial. Journal of Society of Anesthesiologists of Nepal 2016;3(1):28-31.

14. Rachit B, Nanda HS, Mahesh K. Evaluation of Dexmedetomidine- $0.5 \mu \mathrm{g} / \mathrm{kg}$ and $1 \mu \mathrm{g} / \mathrm{kg}$ in Blunting the Responses to Laryngoscopy and Intubation. 